\title{
Erratum to: Endometrial Cancer
}

\author{
Mariana Horta and Teresa Margarida Cunha
}

Erratum to: Med Radiol Diagn Imaging

DOI 10.1007/174_2016_84

The publisher regrets that incorrect affiliation details were included in the published version for authors Mariana Horta and Teresa Margarida Cunha.

The correct affiliation details are provided below.

Mariana Horta $(\square)$

Serviço de Radiologia,

Instituto Português de Oncologia de Lisboa Francisco Gentil,

Rua Prof. Lima Basto, 1099-023 Lisboa, Portugal

mariana.sf.horta@gmail.com

Teresa Margarida Cunha

Serviço de Radiologia,

Instituto Português de Oncologia de Lisboa Francisco Gentil,

Rua Prof. Lima Basto, 1099-023 Lisboa, Portugal

tmargarida@gmail.com

The updated original online version for this chapter can be found at

DOI 10.1007/174_2016_84 